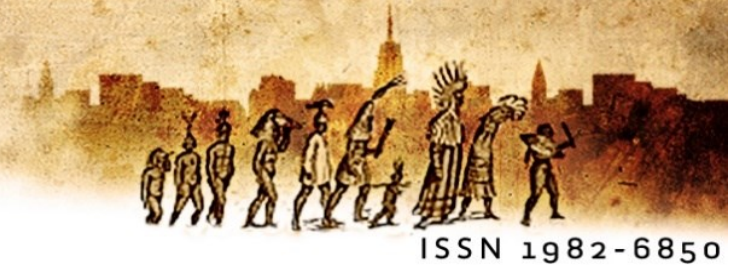

\title{
Eugenio Coseriu et l'école linguistique de Tübingen
}

\section{Eugenio Coseriu e a escola linguística de Tübingen}

Eugenio Coseriu a fait école. Ceux qui font partie de l'école et qui ont fait, sous les yeux critiques, mais bienveillants du maitre, les premiers pas dans la linguistique, se félicitent d'avoir été à bonne école.

(Harald Tuhn)

Eugenia Bojoga ${ }^{i}$

Université Babes-Bolyai, Cluj-Napoca, Roumanie

Resumé: Trois aspects essentiels sont à prendre en compte lorsqu'on parle d'une "école" dans l'expression "faire école": la dimension active de l'enseignement, l'amour pour le Maître et la capacité de travail du Maître. Dans ce travaille, nous essayons de voir dans quelle mesure ces trois conditions sont à retrouver dans l'école linguistique fondée par Eugenio Coseriu à l'université de Tubingen. Nous nous servirons, dans ce but, de plusieurs données "extérieures" des entretiens que nous avons eu la chance de réaliser avec quelques-uns de ses anciens disciples et des évocations du Maître parues en revue ou en volume, à diverses occasions.

Mots clés: École linguistique; Histoire de la Linguistique; Linguistique coserienne.

Resumo: Três aspectos essenciais são levados em conta quando se fala de "escola" na expressão "fazer escola": a dimensão ativa de ensino, o amor pelo Mestre e a capacidade de trabalho do Mestre. Neste trabalho, nós tentaremos observar em que medida essas três condições se apresentam na escola linguística fundada por Eugenio Coseriu na Universidade de Tubinga. Para atingir esse objetivo, nós utilizaremos dados de entrevista que fizemos com alguns de seus antigos discípulos e de evocações sobre ele em diversas publicações.

Palavras-chave: Escola linguística: História da Linguística; Linguística coseriana.

\section{Introduction: Faire école}


Dans un texte emblématique paru en 1981 et intitulé "Faire école" (THUN, 1981), le professeur allemand Harald Thun affiche ouvertement son affiliation au mouvement idéatique lancé par Eugenio Coseriu. Pour définir ce mouvement, Thun fait appel à une expression assez problématique, "école", qu'il conviendra d'expliciter dans les pages à venir. $\mathrm{Si}$, pour le linguiste allemand, l'expression "faire école" est un exemple prototypique de discours répété, cette manière de prendre en compte ce phénomène s'explique par la conception linguistique que Thun venait de s'approprier. Selon celui-ci, cette expression ne renverrait pas seulement au Maître (ou à sa vision idéalisée), mais elle s'étendrait jusqu'à englober tous ses collègues, tout autant de disciples de ce même Maître. Bien conscient de s'inscrire, de la sorte, dans une lignée idéatique regroupant les partisans d'une école linguistique déjà légitimée, Thun n'hésite pas à évoquer la séduction que le linguiste exerçait sur les esprits de ses disciples, une séduction liée à une vocation pédagogique particulière: "E. Coseriu a su garder cette fraîcheur d'idées et cette largeur de vues qui font impression sur son interlocuteur tout comme sur son auditoire et sur son lecteur" (THUN, 1981, p. 327). Ainsi, déjà en 1981, lorsque Coseriu fêtait ses 60 ans, Thun caractérisait l'école inaugurée par son Maître comme un mouvement scientifique homogène, fondé sur une vision cohérente et unitaire sur le langage.

Peu à peu, d'autres hommages vont s'ajouter à cet hommage inaugural. Jürgen Trabant insiste, ainsi, sur la dimension acroamatique de la méthode de Coseriu, Reinhard Meisterfeld en examine les fondements scientifiques, Heidi Aschenberg s'intéresse à sa dimension pédagogique tandis que Johannes Kabatek s'attaque aux manuscrits restés non publiés du Maître. Jörn Albrecht prend en compte la manière dont chaque génération de disciples a entendu assimiler et compléter le paradigme scientifique avancé par Coseriu (ALBRECHT, 2003). Nelson Cartagena résume de la manière suivante la portée de son apprentissage auprès du Maître:

Pour moi, l'école linguistique de Tübingen représente tout ce que j'ai appris auprès d'Eugenio Coseriu : la somme de ses connaissances, son parcours didactique, les cours et les séminaires qu'il a donnés au long des années et que j'ai fréquentés avec le plus vif intérêt (voire avec une passion grandissante), sa vision linguistique et le cercle de disciples qui se sont formés autour de lui et qui, chacun de son côté, s'est consacré ensuite à un aspect particulier de l'édifice théorique bâti par le Maître. Certains d'entre nous nous sommes tournés vers la grammaire ou vers la sémantique, d'autres ont préféré la sémiotique ou la traduction. Il y a, parmi nous, des spécialistes en espagnol ou en portugais, en roumain ou en italien, en catalan ou en français, en galicien ou en occitan. Au-delà des créneaux 
étroits de nos spécialisations, l'enseignement de Coseriu est celui qui nous réunit tous et qui nous donne une identité commune [...]. Même ceux qui ne veulent pas le reconnaître ouvertement ont subi l'influence de ses concepts, de sa manière particulière d'interpréter les faits linguistiques, bref, de toute sa démarche scientifique. (BOJOGA, 2011, p. 95)

Vingt-sept ans plus tard, en 2008, la même expression, "faire école" réapparaît sous la plume de Claudia Mejia Quijano dans son ouvrage, Le cours d'une vie. Portrait diachronique de Ferdinand de Saussure (OUIJANO, 2008). Le titre de I'un des sous-chapitres du livre, reprenant cette même expression ("faire école") témoigne de l'importance accrue prêtée par l'auteur à ce type de phénomène. Trois aspects essentiels sont à prendre en compte lorsqu'on parle d'une "école" et l'auteur les examine tour à tour dans une analyse approfondie de I'héritage laissé par Ferdinand de Saussure. II s'agit, tout d'abord, de la dimension active de l'enseignement dispensé. Le Maître est un penseur qui ne fait pas que transmettre de simples informations: il possède, à son tour, une doctrine personnelle. Reproduit par Claudia Mejia Quijano, Antoine Meillet avance les affirmations suivantes à propos de son ancien maître:

Ferdinand de Saussure était, en effet, un vrai maître: pour être maître, il ne suffit pas de réciter devant des auditeurs un manuel correct et au courant; il faut avoir une doctrine et des méthodes et présenter la science avec un aspect personnel. Les enseignements particuliers que l'étudiant recevait de Ferdinand de Saussure avaient une valeur générale, ils préparaient à travailler et formaient l'esprit; ses formules et ses définitions se fixaient dans la mémoire comme des guides et des modèles. (QUIJANO, 2008, p. 253)

Le deuxième aspect concerne l'amour pour le Maître. La fondation d'une école et la capacité de faire ressurgir, dans les esprits des disciples, la passion pour la discipline enseignée dépendent en grande mesure de la manière dont le Maître est aimé, admiré, respecté et, surtout redouté. Voilà ce qu'en dit Murat, cité aussi par Quijano (2008, p. 254):

II y [à l'École pratique des hautes études] enseigna pendant une dizaine d'années avec un éclat et une autorité incomparables et, parmi tant de maîtres éminents, fut l'un des plus écoutés et des plus aimés. Nous admirions dans ses leçons l'information large et solide, la méthode rigoureuse, les vues générales alliées au détail précis, la parole d'une clarté, $d^{\prime}$ une aisance et d'une élégance souveraines.

Enfin, le troisième aspect insiste sur l'extraordinaire capacité de travail du Maître: la ténacité du Maître devrait servir d'exemple à tous ses élèves. 
Essayons, maintenant, de voir dans quelle mesure ces trois conditions sont à retrouver aussi dans l'école fondée par Eugenio Coseriu. Nous nous servirons, dans ce but, de plusieurs données "extérieures" des entretiens que nous avons eu la chance de réaliser avec quelques-uns de ses anciens disciples et des évocations du Maître parues en revue ou en volume, à diverses occasions.

\section{La dimension active de l'enseignement}

Pour qu'il y ait École, la première condition à remplir, selon Quijano (2008), exige que le Professeur ne soit pas un simple passeur de savoirs scientifiques. Celui-ci doit promouvoir, en même temps, une vision critique de la matière enseignée, décantée dans une conception ou une vision personnelle. Cette vision originale est censée offrir aux éventuels disciples un appui, un repère théorique et méthodologique indispensable auquel les disciples vont se rapporter chaque fois qu'ils s'attaqueront à des questions liées à ce domaine particulier de la connaissance humaine.

Dès son arrivée à l'Université de Tübingen, Coseriu mit à la disposition de ses étudiants non seulement des cours consacrés aux particularités des diverses langues romanes, mais aussi un contact profond avec l'histoire de la pensée linguistique et avec ses tendances contemporaines. Coseriu n'hésitait pourtant pas à envisager cette double démarche selon une vision personnelle du langage humain. En s'accordant à voir dans le langage une "activité créatrice", il proposait "un véritable renversement de perspective" (BORCIL $\breve{A}, 1996,43$ ) et posait ainsi les assises d'un processus de reconstruction radicale de la réflexion linguistique antérieure.

L'originalité de la démarche proposée par le jeune professeur serait à rechercher dans le fondement épistémologique de sa conception. Selon le linguiste roumain Mircea Borcila, ce fondement serait constitué de trois prémisses fondamentales. La première prémisse prend en compte la nature créatrice du langage et la possibilité de l'approcher comme un objet autonome du champ scientifique et philosophique. "la démarche de Coseriu suppose une reconstruction théorique de l'autonomie de l'objet de la recherche linguistique dans l'ensemble des sciences humaines contemporaines" (BORCIL $\breve{A}, 1996,44^{-}$ 5). 
La deuxième prémisse épistémologique concerne la place et le statut de la démarche théorique cosérienne dans le cadre des rapports qui s'établissent entre la théorie linguistique et la connaissance empirique.

Pour la première fois, Coseriu définit clairement et rigoureusement le statut légitime, quasi autonome, de la théorie du langage en tant qu'une prémisse essentielle et un cadre général de l'examen linguistique, tout en insistant sur la relation dialectique qui s'établit entre la théorie et l'étude descriptive. (BORCIL $\breve{A}, 1996,44-5$ ).

La troisième prémisse, enfin, une prémisse essentielle pour une appréciation correcte de l'héritage laissé par Coseriu, porte sur le poids de l'histoire de la réflexion linguistique dans son élaboration d'une vision unitaire et intégrale du langage. À son tour, cette vision était censée servir à une meilleure exploration empirique.

L'idée majeure autour de laquelle pivotaient les réflexions linguistiques de l'école de Tübingen concernait l'importance d'une étude intégrale du langage, en général et des différentes langues, en particulier. Les projets et les réalisations de la première génération de disciples sont ancrés dans cette vision générale. En s'appuyant sur l'hypothèse cosérienne d'un possible renversement du principe saussurien selon lequel la langue constituerait l'objet d'étude de la linguistique, les disciples de Coseriu avancent tous la possibilité, voire la nécessité d'une perspective différente:

No hay que explicar el hablar desde el punto de vista de la lengua, sino viceversa. Ello porque el lenguaje es concretamente hablar, actividad, y porque el hablar es más amplio que la lengua: mientras que la lengua se halla toda contenida en el hablar, el hablar no se halla todo contenido en la lengua. En nuestra opinión, hay que invertir el conocido postulado de F. de Saussure: en lugar de colocarse en el terreno de la lengua, hay que colocarse desde el primer momento en el terreno del hablar y tomarlo como norma de todas las otras manifestaciones del lenguaje (inclusive de la lengua). (...) Desde nuestro punto de vista, el estudio de la lengua es estudio de un aspecto del hablar, que no es abstracto ni exterior al hablar mismo y que, naturalmente, es fundamental, pues el hablar es siempre histórico: es siempre hablar una lengua. (COSERIU, 1967, p. 287)

Les étudiants de l'université allemande ne manquèrent pas de saisir la nouveauté apportée par les cours et les séminaires du jeune professeur d'origine roumaine. Rudolf Windisch affirme ainsi:

Bien qu'encore novice dans le domaine de la linguistique romane, [...] je me suis aussitôt rendu compte de la haute tenue scientifique de ses cours, de la nouveauté de l'information apportée et de l'originalité de son interprétation des faits linguistiques. [...] Nous avons choisi les cours de Coseriu pour la simple raison que nous (mes collègues et moi) étions 
conscients que ce n'était que lui qui pût nous faire apprendre la linguistique avec rigueur et professionnalisme. On avait déjà suivi des cours de linguistique romane non seulement en Allemagne, mais aussi en France et en Italie. Or, par rapport à ce qu'on connaissait déjà, Eugenio Coseriu nous a paru tout à fait exceptionnel. Sa formation pédagogique et théorique mise à part, il était le plus grand savant dans le domaine de la linguistique romane. Du moins, c'était notre impression. (BOJOGA, 2001, p. 31)

De sa part, le professeur Wolf Dietrich appréciait chez Coseriu la "variété thématique" de ses cours:

Le professeur Coseriu nous avait tous impressionnés grâce à la variété thématique des cours qu'il nous proposait dès le début. À part ses cours magistraux de linguistique ou de linguistique romane, il avait des séminaires de portugais, de français, d'espagnol, de sarde, de provençal, de catalan ou d'histoire de la langue roumaine. De plus, sa vision théorique des phénomènes linguistiques était extrêmement large. (BOJOGA, 2001a, p. 83)

Lors de l'arrivée de Coseriu à l'Université de Tübingen, Horst Geckeler venait de finaliser son mémoire de maîtrise sous la direction du professeur Wandruszka. II remarqua aussitôt la rigueur de la méthode de Coseriu de même que sa perspective très ample sur les phénomènes de nature linguistique:

La rigueur de la linguistique promue par Eugenio Coseriu, la rigueur théorique de sa doctrine tout comme sa rigueur méthodologique ont été pour nous une nouveauté absolue. Nos anciens professeurs avaient suivi plutôt une formation historiciste au sens où ils faisaient de la linguistique traditionnelle ou comparée. Sans doute, il y avait des noms célèbres dans la linguistique romane comme, par exemple, ceux d'E. Gamillscheg ou de G. Rohlfs. Pourtant, ils ne semblaient pas voir l'intérêt d'étudier la linguistique théorique contemporaine; ce qui les préoccupait, c'était l'examen de quelques aspects particuliers des langues romanes. [...] Par contre, Eugenio Coseriu nous a impressionnés dès le début grâce au fait qu'il avait sa vision particulière non seulement en matière de sémantique, mais aussi en matière de linguistique générale. II n'était pas un professeur à ne faire que transmettre des informations à ses disciples, il avait une doctrine personnelle rigoureusement fondée et très bien construite. (BOJOGA, 2010, p. 23)

Selon les témoignages de ses disciples, pendant ses premières années d'activité à I'Université de Tübingen, Coseriu commençait son cours de linguistique générale par une introduction au structuralisme, tout en virant par la suite dans la linguistique générative et transformationnelle. Il donnait aussi des cours d'histoire de la philosophie du langage, allant de l'Antiquité jusqu'aux temps modernes. Les cours magistraux étaient pour la plupart 
dispensés en allemand et ils étaient ouverts également à des étudiants en études slaves ou en études germaniques.

Coseriu enseignait avant tout des cours de linguistique générale et de linguistique romane, ayant passé et obtenu son habilitation (venia legendi), c'est-à-dire son droit d'enseigner les deux disciplines. Quelle était sa méthode ? En règle générale, il choisissait un thème provenant soit du domaine de la linguistique générale, soit du champ de la linguistique romane, et il ouvrait un débat sur le sujet proposé. Les thèmes abordés étaient pour le moins variés, déconcertant parfois les étudiants plus "sages" et enthousiasmant les "initiés" passionnés. Coseriu mêlait ainsi la théorie à la recherche empirique, la description concrète des faits linguistiques à une vision conceptuelle extrêmement rigoureuse.

Harald Thun remarquait, à son tour, cette particularité de l'enseignement donné par Eugenio Coseriu, tout en avouant sa déception initiale liée à l'étude de la linguistique:

Tout au début, la linguistique romane m'a semblé assez ennuyeuse. Si c'était cela, la philologie, me suis-je dit, il valait mieux que je change de spécialisation. Mais, une fois parvenu dans la proximité de Coseriu, je me suis rendu compte que la linguistique pouvait être bien différente de celle que je croyais être et qu'elle pouvait s'avérer, en effet, séduisante. En quoi consistait cette différence-là? Eh bien, pour Coseriu il n'y avait pas de théorie purement linguistique sans qu'elle s'appuie sur les langues concrètes, sur la réalité linguistique. Ma plus grande 'révélation' visait, peut-être, la constatation que Coseriu faisait des réflexions en s'ancrant dans la langue, dans les faits linguistiques concrets, qu'il ne se plaçait point au-dessus d'eux'1.

Les séminaires et les cours spéciaux touchaient à des questions ponctuelles concernant l'espace linguistique roman tout entier et ils étaient donnés dans la langue abordée au séminaire. À cette époque-là, Eugenio Coseriu était le seul professeur de toute I'Allemagne qui fit de la linguistique romane au sens large du terme. II ne se focalisait pas sur une langue romane particulière comme, par exemple, le français mais se penchait sur toutes les langues romanes (de l'espagnol jusqu'au roumain), y compris les langues disparues comme le dalmate. Gunter Narr se rappelle, ainsi :

À l'époque il a fallu qu'on étudie d'un bout à l'autre le seul ouvrage consacré au dalmate qui ait existé en Allemagne: c'était Das Dalmatische de Mateo Bartoli. Certes, Coseriu n'exigeait pas qu'on apprenne le dalmate pour le parler. De la même façon, on n'était pas obligé d'apprendre le sarde ou le mégléno-roumain afin de converser dans ces idiomes-là. Ce que le

\footnotetext{
${ }^{1}$ Entretien avec le professeur Harald Thun de l'Université de Kiel, réalisé par Eugenia Bojoga, en manuscrit.
} 
Professeur avait en vue, c'était de nous offrir un panorama sur ces langues, sur les différences historiques de même que sur les ressemblances structurales qui existaient entre la Romania occidentale et la Romania orientale. C'était une chose formidable pour nous, qui élargissait notre horizon d'une manière considérable ! [...] Bref, les cours de Coseriu étaient si intéressants et si novateurs que peu de temps après tous les professeurs allemands étaient au courant qu'il y avait, à I'Université de Tübingen, un Professeur étranger d'origine roumaine qui était à la fois un linguiste éminent et un romaniste redoutable. (BOJOGA, 2011a, p. 11)

Un autre aspect qui a impressionnés Horst Geckeler et ses collègues c'était le fait qu'au cours d'une même journée le Professeur pouvait enseigner en plusieurs langues:

En italien, en espagnol, en portugais ou bien, en roumain. S'il devait donner un cours sur une langue particulière, il le faisait dans cette langue même. Or c'était quelque chose d'inouï, aucun de nos anciens professeurs ne l'avait fait jusque-là ! Par exemple, j'admets que le professeur Wandruszka était un fin connaisseur des langues romanes mais il n'a enseigné, toute sa vie durant, qu'en allemand. II est possible qu'à l'époque il n'y ait pas encore eu en Allemagne la tradition - que Coseriu a par la suite imposée, de donner des cours dans la langue qui faisait l'objet d'étude. La linguistique française était enseignée en français, l'histoire du portugais en portugais et ainsi de suite. (BOJOGA, 2010, p. 23)

Ce qui revient dans tous ces témoignages appartenant aux anciens étudiants de I'Université de Tübingen, c'est une idée commune voyant dans le linguiste roumain un véritable esprit novateur. Pour Gunter Narr, Coseriu avait le courage de s'engager sur des voies nouvelles et d'y emmener aussi ses étudiants:

Du moins, c'est ainsi qu'on le percevait à l'époque. Mais je pense que c'était l'avis général, partagé aussi par des collègues plus âgés, c'est-à-dire par des professeurs venus des universités voisines (de Stuttgart, de Constance ou de Fribourg) pour prendre part à nos cours. [...] Coseriu proposait une multitude de cours basés sur des thématiques nouvelles, entièrement inédites et nous, ses étudiants, on en était ravis. Ainsi sommes-nous devenus en peu de temps les disciples de Coseriu et les partisans acharnés de ses conceptions. (BOJOGA, 2011a, p. 12)

L'enseignement proprement dit présentait, pour Coseriu, deux dimensions fondamentales : une dimension créatrice et une dimension humaniste. La complicité tacite existant entre le Professeur et ses disciples facilitait les échanges: le professeur savait comment motiver ses étudiants, il connaissait leurs penchants et cherchait à assouvir toutes leurs attentes. L'empathie dont il était capable rendait le processus d'enseignement plus authentique. Si B. Laca voyait dans cette capacité de prendre en compte les intérêts des 
étudiants un signe de son caractère "généreux", pour H. Aschenberg, il s'agissait là d'une marque de son ouverture intellectuelle vers l'autre, vers l'altérité.

Il est tout à fait vrai que, dans sa qualité de Maître, Coseriu a exercé une grande influence, voire une véritable fascination sur les esprits de ses étudiants. Tout en leur ouvrant la voie vers la connaissance de la linguistique, il leur ouvrait aussi la voie vers la connaissance de soi-même. Comment l'étude de la linguistique romane pouvait-elle capter l'intérêt des étudiants? Comment devient-on passionné de l'étude du langage? Ce sont autant de questions que le Professeur avait formulées pour ses disciples, en les incitant à en trouver la réponse par eux-mêmes.

Pour Coseriu, tout enseignement véritable était un enseignement interactif, possédant une dimension dialogique inhérente. Partisan de la paideia classique, il ne cessait de rappeler le double sens du mot formation dans l'Athènes antique de même que dans la philosophie allemande traditionnelle. La formation, selon Coseriu, était Bildung: "Ses cours n'étaient jamais une simple introduction à des concepts théoriques ou à des théories étrangères", se souvient Manfred Ringmacher.

Ils nous permettaient à pénétrer dans la pensée même du Maître, dans la linguistique d'Eugenio Coseriu. [...] Nous, ses étudiants, nous avions chaque fois l'impression d'entrer dans un univers fascinant - l'univers de la linguistique véritable, où tout a déjà été mis au point - et de le faire avec une aisance particulière, grâce aux indications du Professeur. Le cours magistral commençait presque toujours par la présentation d'un point de vue contraire à la vision du Maître, que celui-ci expliquait et, ensuite, confronter impitoyablement au sien. Ce qui en résultait à la fin, c'était un "édifice théorique" fort convaincant. [...] Les conférences de Coseriu étaient tout à fait particulières et leur dimension polémique très prononcée nous attirait, sur le coup, comme un jeu. La vérité, c'est que je n'ai compris que plus tard qu'il s'agissait, en fait, d'un jeu extrêmement intelligent, sérieux et très bien construit du point de vue rhétorique et pédagogique. Au début, le Professeur captait notre attention grâce à toutes sortes de triades, de concepts et de doctrines séduisantes. Mais il était trop intelligent et trop exigeant pour se contenter de s'y arrêter. Il voulait qu'on engage un dialogue avec lui, il voulait qu'on devienne ses partenaires de dialogue $e^{2}$.

Cette dimension dialogique était essentielle pour le Professeur. D'ailleurs, Coseriu avait, lui aussi, remarqué la double direction que prenait ce transfert ou, pour mieux dire, ce partage des savoirs : "Chaque fois qu'on enseigne quelque chose, on apprend autre chose en

\footnotetext{
${ }^{2}$ Entretien avec Manfred Ringmacher réalisé par Eugenia Bojoga, en manuscrit.
} 
retour, on se rend compte de ce qui n'est pas aisément compréhensible, de ce qui reste encore flou ou dont on n'a pas prévu les possibles prolongements". Et Coseriu de continuer : "On devrait comprendre cet apprentissage actif comme un dialogos, une sorte d'objectivation répétée dans l'Autre, accompagnée par un retour constant à soi-même. La pédagogie, tout comme le langage, est une activité créatrice" (MUNTEANU, 1992, p. 5).

Poussé par ce "besoin impérieux de faire part de ses connaissances aux autres", selon la formule de Munteanu (1992) et grâce à ce contact direct avec ses disciples, Coseriu réussit ainsi à poser les assises d'une véritable école. Si devenir le disciple du Maître était un privilège qu'il fallait assumer d'une manière consciente et responsable, le contact avec les étudiants était, pour Coseriu, une condition indispensable pour l'élaboration de ses propres conceptions et recherches. Coseriu privilégiait une démarche plutôt intuitive et, dans ce sens, il avait besoin d'un auditoire pour développer ses idées. Selon Heidi Aschenberg, pendant le cours magistral, le professeur travaillait à son texte tout en invitant ses étudiants à refaire son parcours en sens inverse, en allant du discours prononcé vers l'idée soustendant ce discours.

Trabant (1997) remarquait, à son tour, la complicité intellectuelle existant entre Coseriu et ses étudiants. Dans le sillage de Platon, Trabant était d'avis que seul un échange véritable à travers le dialogue pouvait conduire à un apprentissage efficace; par conséquent, l'enseignement théorique de Coseriu serait à compléter par un enseignement non écrit, oral, assimilé seulement par ceux qui fréquentaient ses cours.

Ainsi ses élèves seraient-ils devenus des akroatai, des personnes qui écoutent et assimilent un enseignement resté dans une certaine mesure acroamatique, au sens où il concernait un public restreint et, parfois, "initié". Pourtant, à l'encontre de Platon, Coseriu n'était pas un ennemi de l'écriture, n'y voyant point une menace ou un obstacle à la recherche de la vérité. En témoignent les nombreux textes que Coseriu avait initialement prévus pour des exposés oraux mais qui ont été, par la suite, publiés par ses disciples sous la forme de supports de cours.

Par voie de conséquence, il n'est pas illégitime d'affirmer ce qui suit.

Les travaux de début mis à part, l'œuvre tout entière de Coseriu est fondée sur la transposition par écrit de ses exposés oraux. Même ses articles dont le caractère oral n'est pas si évident que dans le cas des notes prises au cours, Coseriu les a rédigés en dictant ses idées à un assistant averti qui faisait son doctorat sous sa direction. [...] Pour écrire, Coseriu avait, donc, besoin de parler, ce qui supposait, bien évidemment, qu'il y ait quelqu'un - 
un akroates - qui l'écoute et qui note tout ce qu'il venait d'entendre. C'est dans ce sens-là qu'on peut parler de l'œuvre de Coseriu comme d'une œuvre 'acroamatique', une œuvre appartenant à la fois au professeur et à ses disciples. Ce mécanisme compliqué de transposition écrite d'un discours oral garde souvent la spécificité particulière de la langue de la "proximité affective»: c'est le cas des ouvrages conçus sur des notes d'étudiants. Cette composante 'acroamatique' fait accroître leur puissance. (TRABANT, 2003, p. XI)

Le choix des anciens disciples de Coseriu de faire diffuser les idées de leur Maître en publiant ses volumes témoigne de leur foi dans l'enseignement reçu de Coseriu. L'idée en appartient à Gunter Narr et à Rudolf Windisch, comme ce dernier l'avoue dans une interview.

Vu que j'avais toujours tout noté, à la virgule près, j'ai eu l'idée de transcrire à la machine les cours du Professeur Coseriu. Figurez-vous, le faire avec les moyens techniques dont on disposait à l'époque, lorsqu'il n'y avait même pas d'appareils photo... J'ai donc collationné mes notes et celles de Gunter Narr, je les ai vérifiées, dactylographiées et, enfin, multipliées. C'est ainsi que sont parus Einführung in die strukturelle Linguistik, Einführung in die transformationelle Grammatik et, enfin, Die Geschichte der Sprachphilosophie von der Antike bis zur Gegenwart. Eine Ubersicht. Le succès en étant immense auprès des étudiants de Tübingen, on s'est dit : $E t$ si on diffusait ces cours multipliés dans d'autres centres universitaires aussi? C'est ainsi qu'on a commencé à faire de la publicité auprès d'autres universités allemandes et on a reçu de nombreuses commandes de multiplication. Juste pour vous faire une idée, on avait à un moment donné trois ou quatre commandes à exécuter journellement. Tous les jours on emballait des colis qu'on envoyait ensuite par la poste. (BOJOGA, 2001, p. 31)

Cette démarche qui n'est pas sans rappeler celle de Charles Bally, d'Albert Sechehaye et d'Albert Riedlinger. Les cours ainsi transcrits ont été diffusés dans toute I'Allemagne. Gunter Narr expliquerait leur succès.

[les cours] venaient de combler un manque dans la bibliographie académique de cette époque-là. II n'y avait aucune étude dans ce domaine alors que les étudiants avaient besoin de manuels et d'un support bibliographique à la fois bien fondé et moderne. Dans ce sens, Geschichte der Sprachphilosofie a constitué une nouveauté absolve. II n'y avait aucun cours universitaire sur ce sujet, d'autant moins un manuel! Deuxièmement, les volumes n'étaient pas chers, ils étaient à la portée de $n^{\prime}$ importe quel étudiant. [...] D'un coup, le nom de Coseriu est devenu célèbre dans toutes les universités allemandes. Figurez-vous, à cette époque-là (on était en 1966 ou en 1967) le Professeur n'avait encore publié que très peu en allemand. Ses écrits fondamentaux étaient rédigés en italien ou en espagnol tandis que ses études de sémantique étaient en français. Grâce aux volumes que nous venions de faire paraître en allemand, il était 
possible pour tout le monde d'accéder enfin à l'œuvre et à la doctrine de Coseriu. (BOJOGA, 2011a, p. 11)

Cette idée de faire multiplier les notes de cours a été vite reprise et mise en œuvre par les autres disciples de Coseriu. Dieter Kastowsky, Hansbert Bertsch et Jörn Albrecht font ainsi paraître, tout à tour, Strukturelle Semantik, Das romanische Verbalsystem et, respectivement, Textlinguistik. Un peu plus tard, en se fondant toujours sur ses notes prises aux cours, Heinrich Weber fait paraître Sprachkompetenz tandis que Rienhard Meisterfeld conçoit son premier tome de l'ouvrage Geschichte der romanischen Sprachwissenschaft, à savoir Von den Anfängen bis 1492.

La cause est entendue: en s'impliquant activement dans la publication et dans la diffusion de l'œuvre de Coseriu, ses disciples ont participé, sans le savoir peut-être, à l'édification de l'École linguistique de Tübingen.

\section{Le Maître suscite l'admiration et le respect}

La deuxième condition indispensable à l'existence d'une École de linguistique est, selon QUIJANO (2008), I'affection des disciples pour leur maître. Véritable axis mundi de l'univers intérieur d'un courant idéologique, le Maître doit être respecté, admiré, adoré et redouté. II s'agit donc d'une richesse de sentiments que le Maître est susceptible d'éveiller chez ses disciples. Autrement dit, nous entrons dans une zone lexicale très vaste : éprouver de la sympathie, traiter avec du respect et de l'affection, témoigner un profond sentiment d'affection et de dévovement, avoir de la considération et une profonde admiration à son égard, et même susciter la peur, se faire redouter par ses disciples. Ce que Quijano (2008) ne précise pourtant pas est que, pour pouvoir susciter chez ses élèves/étudiants la passion pour ses idées, le Maître doit se donner lui-même entièrement. De la plénitude de cet état de sacrifice total naît dans leur âme la passion pour l'étude du langage, propre à engendrer ensuite de l'affection à l'égard de l'"agent de l'action". Et l'agent qui produit la connaissance n'est autre que le Maître.

En ce qui concerne l'École de Tübingen, nous croyons qu'elle n'aurait pas pu exister en l'absence d'une sympathie réciproque et implicitement, du respect à l'égard de leur maître. Les futurs disciples avaient d'abord constaté qu'Eugeniu Coseriu leur proposait une nouvelle méthode d'enseignement, qu'il connaissait et utilisait beaucoup de langues 
étrangères, qu'il avait une ouverture remarquable vers d'autres horizons culturels, et qu'il avait une approche originale du langage, en un mot, qu'il était différent.

Dans cet état d'esprit plein d'enthousiasme, les étudiants comparaient Coseriu à d'autres professeurs de linguistique romane. Dans cette équation, les termes de comparaison sont différents, en fonction de la génération de chaque disciple. Pourtant, ceux dont les noms reviennent souvent sont E. Gamillscheg, Gerhard Rohlfs, Mario Wandruszka, Hans Helmut Cristmann, à côté d'autres. G. Narr déclare, contrairement à Ernst Gamillscheg et à Gerhard Rohlfs:

Eugenio Coseriu apportait de l'air frais et une nouvelle méthode ». II avait ainsi «élevé à un niveau supérieur l'étude des langues romanes à I'Université de Tübingen, en la fondant sur une base philosophiqueconceptuelle très large, ce qu'on n'avait pas fait jusque-là. C'est la raison pour laquelle beaucoup d'étudiants venant d'autres universités d'Allemagne ont commencé à se transférer dans notre université, motivés par la renommée de Coseriu. (BOJOGA, 2011a, p 12).

H. Geckeler, qui avait étudié sous la direction de Wandruszka, apprécie sa diversité thématique, l'extension permanente de ses domaines d'intérêt et son ouverture vers d'autres horizons, insoupçonnés et inconnus aux étudiants allemands de l'époque. Avant l'arrivée de Coseriu à l'Université de Tübingen, aucun de leurs professeurs ne leur avait parlé de l'espagnol d'Amérique du Sud ou du portugais brésilien.

Certes, nous savions tous que l'espagnol et le portugais étaient parlés aussi ailleurs que dans la péninsule Ibérique, mais pas plus. Cela s'explique peutêtre par le fait que nos enseignants n'avaient guère voyagé... ils faisaient partie d'une autre génération. Un voyage en bateau en direction de l'Amérique du Sud durait à peu près six semaines... Or Coseriu, par la vaste thématique de ses cours et séminaires, avait considérablement élargi le champ d'investigation des langues romanes. L'espagnol et le portugais d'Amérique sont devenus dorénavant des domaines récurrents dans notre activité didactique et scientifique. Par ailleurs, en se dirigeant vers l'espagnol et le portugais d'Amérique du Sud, Coseriu a facilité la réception de la littérature hispano-américaine dont il avait une excellente connaissance, à notre grande surprise. Nos professeurs ne possédaient pas un tel savoir, parce qu'ils n'avaient pas son ouverture d'esprit et qu'ils se limitaient à la linguistique, et encore à la linguistique traditionnelle. (BOJOGA, 2010, p. 58)

Wolf Dietrich, qui avait étudié auparavant à l'Université de Münster, ensuite à Montpellier, en France, avait des termes de comparaison différents. Il déclare que le Prof. Coseriu l'avait impressionné d'abord par la diversité thématique des cours qu'il proposait. 
Un vaste répertoire de cours optionnels dans toutes les langues romanes, ce que nos professeurs n'avaient jamais fait avant. Je me rappelle que, à part les cours magistraux de linguistique générale et de linguistique romane qu'il avait l'obligation de donner, Coseriu avait l'habitude de nous offrir chaque semestre un nouveau cours, que ce soit un cours spécial sur le portugais ou le sarde, sur le provençal ou le catalan, sur le latin vulgaire et les premières différenciations dans les langues romanes, sur le système verbal ou la typologie des langues romanes. En plus, comme il était un fin connaisseur des littératures écrites dans ces langues, il proposait des cours intitulés de façon générique Commentaires de textes littéraires, pour chaque langue romane, où il proposait une interprétation critique-philologique des textes les plus représentatifs. (BOJOGA, 2001a, p. 85)

Il n'y a pas que H. Geckeler, W. Dietrich, G. Narr, J. Albrecht, etc. qui peuvent s'exprimer légitimement sur la différence entre Coseriu et les autres enseignants, mais aussi les disciples des générations suivantes. Ce qui séparait, par exemple, $\mathrm{E}$. Coseriu et $\mathrm{H}$. Helmut Christmann était, selon M. Ringmacher, que "le professer Coseriu avait son propre système - sa théorie linguistique - en lequel il croyait, alors que le Professeur Christmann n'acceptait même pas une telle possibilité" (BOJOGA, 2011, p. 19).

Les cours enseignés par Coseriu, son message scientifique, ses qualités d'enseignant ont été remarqués non seulement par ses étudiants allemands, mais aussi par des étudiants d'autres pays. Ana Agud se souvient qu'elle avait fini ses études et qu'elle voulait écrire une thèse de doctorat sur la description structurale des cas en grec et en latin (en 1971), mais que les professeurs de I'Université de Salamanque trouvaient ce sujet difficile et relativement sans perspective. Sa chance a été qu'au même moment, Eugenio Coseriu fût arrivé à Salamanque, invité par le prof. E. de Bustos Tovar pour donner une série de conférences sur la sémantique structurale. "En assistant à ces cours-là, je me suis tout de suite aperçue de l'envergure scientifique et philosophique de sa vision - que je trouvais d'abord fort intéressante - et des perspectives qu'elle ouvrait" (BOJOGA, 2003, p. 32).

Nelson Cartagena avait eu la même impression quand il avait connu Coseriu, alors qu'il était un jeune étudiant au Chili.

Les cours et tout le message de Coseriu apportaient une onde de fraîcheur à l'Université de Santiago de Chili, c'est pourquoi ils m'ont produit une impression extraordinaire, et sa personnalité m'a véritablement fasciné. Au-delà des aspects d'une nouveauté absolue de ses enseignements, Coseriu m'a impressionné par sa connaissance parfaite de plusieurs langues. Pendant ses conférences, il reproduisait de mémoire des extraits en latin, en grec ancien, en allemand, en français, en italien, etc. J'ai remarqué également le caractère systématique de ses 
exposés, sa créativité et son intelligence hors pair. Comme j'étais tout jeune, moi, j'en étais complètement fasciné. (BOJOGA, 2011, p. 98)

Les disciples témoignent qu'Eugenio Coseriu était pour eux un Professeur séduisant, avec un charme personnel particulier, très exigeant et particulièrement érudit. Les jeunes de Tübingen qui avaient eu l'occasion de participer à ses cours ou de l'écouter à l'occasion de ses séminaires avaient remarqué l'ampleur de ses connaissances, l'envergure de sa vision sur le langage, la modalité interactive de leurs rencontres hebdomadaires et son charisme. Autrement dit, ils se sont rendu compte que Coseriu était le Professeur qu'ils cherchaient intuitivement, le Professeur dont ils avaient besoin pour étudier, pour se former. La plupart de ses disciples ont eu la chance d'entrer en contact avec le Professeur, pendant leurs études à Tübingen, d'autres sont venus exprès pour le rencontrer. Ce fut le cas de Jürgen Trabant, qui s'est transféré de I'Université Libre de Berlin, de Rudolf Windisch qui est venu de I'Université de Bonn et de Brigitte Schlieben-Lange qui avait quitté I'Université de München pour étudier avec Coseriu. D’autres étudiants sont venus d'Amérique du Sud. Ce phénomène de «migration » à Tübingen se manifeste également chez les disciples de Coseriu des générations ultérieures.

Une autre catégorie de disciples ont découvert Coseriu en lisant d'abord ses travaux. L'exemple typique est celui d'Harald Thun, qui, étant originaire du nord de l'Allemagne, avait commencé ses études à l'Université Christian-Albrecht de Kiel. Voici la suite:

Dans le premier semestre j'ai lu un texte d'Eugeniu Coseriu, Structure lexicale et enseignement du vocabulaire, qui m'a beaucoup plu parce qu'il combinait la théorie et l'analyse des faits de lexique. Or, c'est à cette époque-là que notre professeur de linguistique romane a quitté Kiel. Alors je me suis dit que je pourrais aller dans une université où il y avait déjà un professeur renommé, avec qui je pourrais apprendre sérieusement la linguistique romane. Voici comment je suis arrivé à Tübingen. C'était en 1969. (THUN, 1981, p. 325)

Dans le cas d'Heidi Aschenberg, une forte sympathie s'est manifestée dès la première lecture. Une sorte d'empathie entre le lecteur et l'auteur. II est certain que les textes de Coseriu, bien au-delà des mots, lui ont transmis quelque chose. Après des lectures sérieuses et rigoureuses, elle prendra la décision:

En 1973, lorsque j'étais étudiante à l'université de Bonn, j'avais obtenu une bourse d'un semestre à Paris. C'était à l'époque où je venais de découvrir Coseriu et où je lisais ses articles avec beaucoup d'intérêt et d'acribie. Je me suis alors rendu compte que, si j'étudiais sous sa direction, je m'occuperais non seulement de la philologie romane, mais 
aussi de la philosophie du langage. Étant donné que les deux disciplines m'attiraient autant, en découvrant Eugenio Coseriu, j'ai en fait découvert le professeur dont j'avais besoin. Mes intérêts, associés à mes études philosophiques, ne s'orientaient pas vers la linguistique empirique (...), mais plutôt vers une perspective interdisciplinaire, disons, vers la rencontre de la linguistique romane et de la critique littéraire ou vers la conjonction entre la linguistique romane et la philosophie. J'ai donc reconnu en la personne de Coseriu le Maître idéal pour mes préoccupations. Surtout après avoir lu Geschichte der Sprachphilosophie, les deux volumes édités par Narr et Windisch, je fus cent pour cent persuadée que c'était lui le professeur que je cherchais avec tant de ferveur. C'est pourquoi, en rentrant de Paris, la première chose que j'ai faite a été de me transférer de l'Université de Bonn à celle de Tübingen³.

Pour Donatella di Cesare, le "médiateur" a été Tullio di Mauro, son professeur de philosophie du langage de l'Université de Rome, qui lui avait indiqué une référence obligatoire pour son mémoire de licence, Geschichte der Sprachphilosophie von der Antike bis zur Gegenwart, en lui suggérant d'aller à Tübingen. Voici son témoignage:

Mon mémoire de licence à la main, je suis allée voir Coseriu immédiatement après mon arrivée à Tübingen. Il y avait du monde, et j'ai dû attendre. Entre temps, en Italie, j'avais déjà reçu nombre de descriptions à son égard. La plupart n'étaient pas du tout favorables (...). Mon impression a pourtant été tout à fait différente, car je me suis aperçue tout de suite que son autorité, qui n'avait rien à voir avec l'autoritarisme, était proche du charisme. J'avais vingt-deux ans, mais je n'étais pas effrayée. II m'a dit d'entrer et a commencé à parler un italien impeccable, sans le moindre accent. Je lui ai dit, en me sentant un peu ridicule: "Je viens de Rome". "Pèlerin venant de Rome", a-t-il répliqué, en souriant. Et il a ajouté en sicilien, allusion volontaire à Pagliaro: "Revenez demain".

Le lendemain, lorsque je me promenais à Neuphilologikum de Tübingen, quelqu'un m'a interpellée en me demandant, non sans envie, comment j'avais réussi à déterminer Coseriu à lire intégralement mon mémoire du jour au lendemain. Mais je ne savais pas de quoi il s'agissait. Je suis allée voir Coseriu. Frau Ott, la secrétaire, m'a fait entrer. II m'attendait, mon mémoire à la main, rempli de fiches et d'annotations. C'était vrai, il l'avait lu. Il a commencé par une critique impitoyable, ligne après ligne. II était très concentré, les yeux dans le vide. II parlait avec une précision terminologique et conceptuelle que je n'avais rencontrée chez personne jusque-là. Je me suis dit qu'un jour, j'aimerais parler moi-même de cette façon, et en autant de langues que possible. De Héraclite, il est passé à Platon, puis à Aristote, en indiquant parfaitement les limites de mon mémoire, et en me stimulant à les surmonter.

Ce fut comme s'il avait lu toute la bibliographie auxiliaire pendant la nuit et avait réussi à en faire une magistrale synthèse. Et il conclut, en disant :

3Entretien avec le Prof. Heidi Aschnberg, Université de Tubingen, Allemagne, réalisé par E. Bojoga, en manuscrit. 
"Je dois vous dire quelque chose de très dur. Vous devez renoncer à tout. Vous devez uniquement étudier. Vous devez rester en Allemagne et étudier"4.

Pour les disciples, le fait de rencontrer leur Maître a été décisif. Leurs années de formation n'ont pas signifié uniquement une accumulation de connaissances, mais aussi un véritable apprentissage qui allait les marquer du point de vue intellectuel autant qu'humain. Certains admettent que le fait d'avoir connu Coseriu les a profondément marqués.

Mon apprentissage auprès de Coseriu a été la période la plus intéressante et la plus importante dans ma formation de linguiste... Avec mes collègues et amis, nous avons pu profiter de quelques années fécondes pour notre formation, où nous avons beaucoup discuté, nous avons appris à percevoir une langue de l'intérieur, et, ce qui est le plus important, nous avons appris à réfléchir de façon autonome. (BOJOGA, 2001, p. 35)

Coseriu leur a appris que, pour devenir des linguistes, ils devaient se donner et se sacrifier, s'ouvrir vers autant d'horizons culturels que possible. Les disciples ont assimilé les messages implicites et explicites du Maître. Comme le révèle nos entretiens, le professeur Coseriu leur demandait une attitude sérieuse à l'égard de l'étude, du dévouement, de la persévérance, de l'abnégation et du sacrifice. Exigeant à l'extrême, mais aussi affectueux au niveau de la relation humaine, sa personnalité a exercé la force d'attraction nécessaire pour fasciner les jeunes. Il leur a ouvert une voie vers la connaissance de la linguistique, mais surtout vers la connaissance de soi. Mais avant, dans les séminaires spéciaux, ils ont eu besoin de temps pour s'habituer à un type de critique très dure.

Si quelqu'un présentait un mémoire, il devait subir toutes les conséquences, les remarques et les suggestions d'E Coseriu, ce qu'aucun autre enseignant n'avait fait d'une manière aussi implacable. Le professeur analysait nos affirmations, phrase par phrase et nous demandait quel sens avait tel ou tel syntagme (...). Paradoxalement, cela a stimulé les meilleurs. Par ailleurs, ils pouvaient discuter avec lui à la fin des cours, non seulement aux séminaires. Le Professeur était disponible pour eux à n'importe quelle heure! Ce fut, je crois, la meilleure méthode pour créer ce qu'on a appelé plus tard l'École linguistique de Tübingen. (BOJOGA, 2001, p. 37)

En ce qui concerne ces séminaires pour les étudiants avancés, Manfred Ringmacher déclare avoir beaucoup appris, surtout grâce aux résumés rédigés par le Professeur à

4Entretien avec le Prof. Donatella di Cesare, Université Sapienza de Rome, réalisé par E. Bojoga (en manuscrit). 
propos du mémoire présenté la semaine respective, et dans lesquels il mettait les choses au point.

\begin{abstract}
Coseriu avait le don d'éclairer les choses, de nous les rendre plus accessibles, de nous les présenter sous une forme attractive. C'est une qualité que j'ai toujours admirée chez le Professeur: tu parlais avec lui et d'un coup, il t'expliquait ton propre point de vue, mais d'une manière beaucoup plus claire et nuancée. Un jour, dans un séminaire sur l'histoire des cas, il y avait très peu d'auditeurs, en raison probablement de l'absence de cette catégorie dans les langues romanes. Par conséquent, les romanistes n'avaient pas à s'occuper de cet aspect. Alors il y a eu les membres du département, son assistant, ses doctorants et moi-même. Le résultat a été un des séminaires les plus intéressants dont je puisse me souvenir. J'ai eu le courage de parler de la théorie d'Hjelmslev exposée dans La catégorie des cas. Certes, je ne me sentais pas préparé, mais avec les explications du Professeur, je m'en suis sorti très bien. Avec sa vaste expérience de lecteur des textes d'Hjelmslev, Coseriu m'a encouragé et m'a aidé. C'est ainsi que je le revois dans mon souvenir, avec ses contributions brillantes en situation de dialogue: I'étudiant posait une question et ne recevait pas seulement une réponse, mais une série de réponses à des questions qu'il n'avait pas encore eu le temps de formuler, mais qui existaient dans sa tête ${ }^{5}$.
\end{abstract}

Dans la mémoire de ses disciples, Eugenio Coseriu a été un individu en permanente évolution intellectuelle, motivé par un vif intérêt pour tous les aspects des langues et du langage en général. En plus, il lisait tout ce qui lui tombait sous les yeux, tout ce que les autres lui envoyaient: ses disciples, ses amis, des linguistes de différents pays. N. Cartagena confirme que Coseriu a toujours intégré de nouveaux thèmes et des aspects inédits dans son champ de recherche :

S'il avait une étudiante du Japon, Coseriu commençait à s'intéresser à la grammaire du japonais, s'il avait un doctorant de l'Afrique du Sud, il commençait à se préoccuper des langues africaines et créoles, etc. II était toujours comme ça. (...)

Même si Coseriu lui-même n'a pas écrit une œuvre monumentale unique, il a écrit tant de travaux, tant de livres mémorables à travers ses disciples. Le fait de s'être dédié à ses élèves a ainsi multiplié son œuvre par eux. Son esprit et sa théorie ont été adoptés par tous ses disciples, qu'ils l'admettent ou non. (...) Je me souviens d'un geste de notre regrettée Brigitte Schlieben-Lange, qui, à l'occasion du $70^{e}$ anniversaire de Coseriu, nous a envoyé à chacun de nous, ceux qui étions présents à cette réunion, une carte postale de remerciements, en les adressant 'À Coseriu, à Heidelberg' et elle me l'a expédiée, 'à Coseriu, à Münster', et elle l'a envoyée à Dietrich et à Geckeler. Et ainsi de suite, 'à Coseriu, à Kiel', et elle l'a envoyée à $\mathrm{H}$. Thun, 'à Coseriu, à Rostock' et elle l'a envoyée à R. Windisch, etc. Elle a envoyé ainsi à chaque disciple de Coseriu une carte postale symbolique.(BOJOGA, 2011, p. 97)

\footnotetext{
${ }^{5}$ Entretien avec Manfred Ringmacher réalisé par E. Bojoga (en manuscrit).
} 
Dans la mémoire de ses disciples, Eugenio Coseriu est comme un Guru hindou, un Maître spirituel moderne, qui pratique toutes les vertus et qui dissipe par la lumière de sa raison l'épaisse obscurité entourant ses disciples. Il se conduit dignement et indépendamment, et il a une âme de père pour chacun de ses disciples. Par sa sagesse, il brille comme le soleil au milieu des nuages denses de l'ignorance qui l'entoure. Voici le témoignage d'Ana Agud.

Je n'ai jamais connu, ni directement ni indirectement, un individu d'une culture aussi vaste et aussi exacte que celle d'Eugenio Coseriu. Ses connaissances ne concernaient pas uniquement ses compétences en langues (modernes et classiques), incomparables à celles des autres linguistes, mais aussi une connaissance profonde de leurs littératures, lues en original. En plus, il avait une connaissance de première main pratiquement de toute la bibliographie linguistique européenne de I'Antiquité jusqu'à présent. Ses lectures en philosophie et en esthétique étaient complètes. Il avait une culture impressionnante dans d'autres domaines également, et dans d'autres sciences: histoire, mathématiques, sciences de la nature, psychologie, sciences sociales, etc. Eugenio Coseriu a probablement été le dernier 'génie universel' de l'histoire européenne. [...] Les théoriciens de la littérature ont l'habitude de dire aujourd'hui que ce concept - celui de 'génie' - issu du romantisme est déjà obsolète. Moi, je les contredis, en disant non, ce n'est pas vrai. Des génies, il en existe... j'en ai connu un! Il est complètement différent de tous les autres et quand on le croise, on l'identifie tout de suite, on le reconnaît. Ce n'est pas seulement une particularité culturelle d'une époque, il existe vraiment des hommes de génie. Eugenio Coseriu en était un. (BOJOGA, 2003, p. 32)

\section{La capacité de travail du Maître}

La troisième condition est étroitement liée aux deux premières - la dimension active de l'apprentissage, le respect et l'admiration du maître: sa capacité de travail. Quijano (2008) estime qu'un vrai mentor doit être doué d'une force de travail hors du commun - comme l'était d'ailleurs F. de Saussure, de sorte que son exemple d'effort soutenu, de ténacité et de persévérance devienne un modèle pour ses élèves.

Les représentants de l'École de Tübingen ont d'abord remarqué la force de travail d'E. Coseriu comme I'un des traits fondamentaux de sa personnalité. Nous savons aujourd'hui qu'il a été productif toute sa vie. Même peu avant sa mort, alors qu'il était gravement malade, il demeurait assis à sa table de travail, lisant et rédigeant ses dernières études. Et pourtant, c'est l'époque de Montevideo - les années 1951-1963 - qui a été la plus prodigieuse de tous les points de vue. C'est de cette époque-là que datent ses études 
fondamentales, Sistema, norma y habla, Forma y sustancia en los sonidos del lenguaje, La geografía linguística, Determinación y entorno, Sincronía, diacronía e historia, etc. Par ailleurs, Coseriu a écrit d'amples études encore inédites, La corección idiomatica et Teoría linguística del nombre propio, dont les manuscrits - disponibles dans son Archive de I'Université de Tübingen - comptent des centaines et des milliers de pages. En même temps, il était professeur à la Faculté de Sciences Humaines de I'Université de Montevideo et à l'Institut Pédagogique où il enseignait la linguistique générale, la philologie classique, l’histoire de la langue italienne, la dialectologie espagnole, etc. En plus, il donnait des cours de Psychologie de l'art et d'Histoire du journalisme italien, disciplines éloignées de la philologie. À un moment donné, il totalisait 48 heures par semaine, " ce qui est aujourd'hui inconcevable ». En l'absence de matériel bibliographique, il préparait pour beaucoup de ses cours des introductions ou des recueils de textes: Textes pour le cours de latin, Introduction à la linguistique, Histoire de la langue espagnole, Textes d'esthétique, etc. Bref, comme l'affirme J. Kabatek, Coseriu menait à l'époque une activité si intense, qu'on peut difficilement imaginer qu'il ait eu le temps de dormir 6 .

Or, connaissant tous ces détails et voyant la capacité de travail d'E. Coseriu, qui se manifestait à Tübingen à travers cette activité particulièrement vaste et diverse, ses élèves s'efforçaient de tenir le rythme, de le suivre. D'ailleurs, ils témoignent qu'il y avait beaucoup de travail et de discipline de lundi à samedi. L'exemple de Jens Lüdtke est significatif à ce propos. En se rendant en fin d'année à Finanzamt (service des impôts) pour déposer sa fiche de revenus pour l'année en cours, il a déclaré qu'il avait travaillé de lundi à samedi (il avait ainsi le droit de bénéficier d'une déduction, en évoquant le trajet parcouru entre sa maison et I'Université). Les fonctionnaires lui ont demandé une attestation qui certifie qu'il travaillait le samedi, parce qu'en Allemagne, on travaillait cinq jours par semaine. J. Lüdtke est allé voir le Professeur, lui a expliqué la situation, et E. Coseriu n’a pas hésité une seule seconde à lui fournir cette attestation.

Il est certain que le Professeur Coseriu a su mobiliser tous ses élèves par son exemple. Certains ont atteint le niveau de tension intellectuelle auquel il était lui-même connecté. Comme l'affirme R. Windisch, «si quelqu'un voulait devenir un disciple de Coseriu, il devait tout au moins essayer de travailler autant que lui ». Conscients de leurs

\footnotetext{
${ }^{6}$ Pour plus de détails sur l'époque de Montévidéo, voir Kabatek et Murguía (1997). , «Die Sachen sagen wie si sind...» Eugenio Coseriu im Gespräch, Narr Verlag, Tübingen 1997.
}

Eutomia, Recife, 30(1): 1-27, Dez. 2021 
propres limites et de l'impossibilité d'atteindre une performance pareille, tout ce qu'ils pouvaient faire était de «se proposer au moins de l'imiter ». Responsables et sérieux, ils se sont entièrement dédiés à l'étude, en assumant l'exigence cosérienne de ténacité et de sacrifice de soi. Ils ont assumé aussi un style de vie quelque peu «spartiate » dans leur activité. C'est vrai, ce fut une véritable provocation, mais les plus puissants ont résisté et ont réussi. Le grand secret résidait dans la capacité du Professeur de les motiver par son exemple de persévérance et d'abnégation.

La capacité de travail de Coseriu était hors du commun, ce qui signifiait qu'il était capable de faire beaucoup plus pour ses disciples que ses collègues, professeurs «normaux». Dans ce cadre, le cercle qu'il a fondé à Tübingen, appelé aussi le Colloque des doctorants, a été une véritable école linguistique. Imposant un niveau théorique très élevé, le Colloque a contribué à leur formation scientifique et intellectuelle en général. Du point de vue du professeur, ce cadre ouvrait la voie à la découverte de la vocation de chaque élève. Du point de vue des disciples, il était la continuation des préoccupations initiées aux cours, un approfondissement rigoureux d'aspects partiellement connus. Pour tous, ce fut un lieu de travail idéal, une manifestation immédiate de leur complicité scientifique. Avant tout, il y régnait un véritable état d'effervescence et une atmosphère authentique de communion intellectuelle.

Le colloque des doctorants a été important parce qu'il leur a permis de se constituer en tant que groupe homogène, malgré la diversité de leurs préoccupations. II y avait non seulement des romanistes, mais aussi des slavistes, non seulement des linguistes, mais aussi des philosophes. Ils se rencontraient chaque samedi, de $9 \mathrm{~h}$ à $13 \mathrm{~h}$, et les séances se déroulaient sans pause.

Chaque participant devait faire un exposé, généralement axé sur la problématique de sa thèse de doctorat, suivi d'un débat. Le professeur intervenait par moments, et faisait un excellent résumé des thèses du doctorant, légèrement modifiées, pour que « tout reçoive un sens plus cohérent ». Ils savaient tous ce que faisaient les autres, ils partageaient souvent leurs trouvailles intellectuelles, leurs lectures, leurs bibliographies et se faisaient des suggestions diverses. La présence du Professeur était tout aussi importante, grâce à ses questions, à ses suggestions, à ses interprétations.

Ludmila Golubzowa, qui venait de soutenir sa thèse sur la stylistique du russe et qui travaillait au département de langues slaves, se souvient avec nostalgie des séances du 
Colloque des doctorants: "les rencontres étaient toujours très intéressantes". Présente à toutes ces rencontres, elle déclare qu'elle n'avait jamais vu Coseriu apporter un manuscrit aux séances du colloque, mais que "ses interventions présentaient pourtant l'essence de la problématique, qu'elles étaient magnifiquement structurées, qu'il savait toujours à quel moment faire une remarque ou un renvoi à un auteur, qu'il citait toujours des phrases entières de la littérature de spécialité, qu'il apportait toujours le bon exemple pour illustrer n'importe quelle idée" (GOLUBZOWA, 2002, p. 77-8).

En ce qui concerne la méthode de travail de Coseriu, ses anciens étudiants racontent qu'il avait l'habitude de dicter ses articles et ses études à ses assistants. Cette dictée était, selon Harald Thun, d'une rigueur exemplaire. Avant de commencer sa dictée, Coseriu pouvait passer beaucoup de temps à réfléchir à ce qu'il allait dire:

Sur toute la feuille de papier il n'y avait qu'une seule ligne écrite, le reste de l'espace étant laissé vide pour des notes ou des ajouts ultérieurs. C'était la première étape du brouillon. II nous disait de laisser des blancs pour y transcrire, plus tard, les citations. Le sujet, quel qu'il fût, devait être traité de la manière la plus exhaustive possible, c'est-à-dire en analysant tous ses aspects jusqu'à atteindre le cœur du problème. Au moment de la rédaction proprement dite, c'était comme une édition critique sur laquelle on pouvait jover. Ce fut pour nous une expérience fort utile... Du moins, dans mon cas, j'ai appris beaucoup de choses. C'est ainsi que j'ai appris comment rédiger un article et cette méthode, je l'emploie de nos jours encore. [...] En ce qui concerne le Professeur, il était extrêmement critique ${ }^{7}$.

Selon une anecdote racontée par J. Kabatek, Coseriu préparait à un moment donné la publication d'un recueil d'entretiens intitulé Die Sachen sagen wie sie sind. Eugenio Coseriu im Gespräch. Et même sur le bon à tirer, Coseriu ne cessait d'envoyer à son éditeur des corrections, des rajouts, des phrases changées ou de courtes listes de fautes. II était constamment à la recherche du mot ou de l'expression juste.

Nous, ceux qui travaillions avec lui ou qui l'aidions à transcrire ses articles, nous connaissions fort bien sa recherche du mot juste. De ce point de vue, on pouvait comparer Coseriu à un poète. D'ailleurs, Thomas Mann n'a-t-il affirmé que pour les écrivains, les efforts impliqués par le fait d'écrire étaient plus grands que pour le reste d'entre nous? (BOJOGA, 2005, p. 17).

Kabatek dit aussi:

Lorsqu'il écrivait un article en allemand, en français ou dans d'autres langues étrangères, Coseriu aimait avoir dans sa proximité un locuteur natif. Pourtant, ce n'était qu'un moyen d'avoir devant lui une sorte de miroir ou d'écho: c'était toujours à lui qu'incombait l'initiative. Pendant

\footnotetext{
7 Entretien avec le professeur Harald Thun de l'Université de Kiel, réalisé par Eugenia Bojoga, en manuscrit.
} 
qu'il dictait ses textes il les regardait aussi ; de cette manière-là, il les avait à la fois dans sa tête et devant ses yeux. Il pouvait, ainsi, se rappeler un mot qu'il avait déjà employé bien des pages avant ou qu'il avait dicté plusieurs heures avant et que la personne qui écrivait sous sa dictée avait complètement oublié.(BOJOGA, 2005, p. 17)

Pour les disciples de Coseriu, il s'agissait d'une méthode à assumer : celle de tout préparer mentalement avant de commencer la rédaction proprement dite d'un travail écrit. Selon leurs propres dires, ce qu'ils avaient appris auprès de leur Maître, ce n'était pas seulement la manière dont on pouvait traiter diverses questions de linguistique ou de philologie romane. Coseriu leur avait offert quelque chose de plus précieux encore, à savoir des méthodes de travail qu'on pouvait adopter à maintes occasions.

L'énergie du Maître était, somme toute, extraordinaire. Kabatek n'omet pourtant pas de remarquer que, parfois, cette énergie pouvait « fatiguer ceux qui se trouvaient dans sa proximité. Il ne comprenait pas toujours qu'on pouvait avoir besoin également d'une pause. » Une fois, se souvient Kabatek, Coseriu devait rattraper un cours. C'était en été et il faisait une chaleur insupportable. Et Coseriu de parler pendant quatre heures, sans aucune pause.

Sa capacité de travail était, en effet, hors du commun ${ }^{8}$. Dans ce sens, même les collègues de Coseriu, professeurs, eux aussi, à l'Université de Tübingen voyaient dans le travail du professeur d'origine roumaine l'expression d'une force surhumaine.

Lorsqu'il devait parler de la capacité de travail de son professeur, Nelson Cartagena utilisait une métaphore significative, en le présentant comme un «homme-orchestre». Pourquoi ce choix stylistique? Pendant ses premières années à l'Université de Tübingen, Coseriu avait introduit l'enseignement de toutes les langues romanes, I'occitan y compris. II y a avait des «lectorats » officiels pour bon nombre d'entre elles et des professeurs associés natifs qui enseignaient des cours dans leur langue maternelle. À son tour, le professeur Coseriu enseignait de nombreux cours sans bénéficier d'une rémunération des heures supplémentaires. Affirme Cartagena:

Il jouait à tous les instruments à la fois, c'est-à-dire il connaissait toutes les langues romanes, sans exception. D'ailleurs, il parlait toutes ces langues-là, ce qui était, pour nous, un modèle vivant de sagesse et d'intelligence. Cet aspect a impressionné à la fois les étudiants et les professeurs allemands.

\footnotetext{
${ }^{8}$ «Eugenio Coseriu, lui, il a été un titan ; il a couvert tous les champs de la linguistique. Tandis que nous, nous sommes des personnes normales, des linguistes normaux et, par conséquent, nous ne maîtrisons qu'un seul domaine de la linguistique. » (J. Kabatek).
} 
Comme on le sait, la tradition allemande en matière de philologie romane est, peut-être, la plus solide au niveau mondial. Et pour autant, un savant si célèbre comme, par exemple, Meyer-Lübke ne parlait pas le français moderne. Une fois, lorsqu'il a été obligé de donner une conférence en France, il a parlé en... ancien français! Ainsi des professeurs comme Eugenio Coseriu ou Mario Wandruszka [...] qui parlaient toutes les langues romanes étaient-ils des exceptions. Pourtant, par rapport à Wandruszka, Coseriu était vraiment un homme-orchestre puisqu'il possédait des compétences actives dans de nombreuses langues. C'était un connaisseur non seulement des langues romanes mais aussi des langues slaves ou des langues germaniques... C'est pourquoi le professeur Coseriu, tout en étant le modèle vivant d'un philologue romaniste complet, était une exception aussi pour les Allemands. (BOJOGA, 2011, p. 101)

Un corollaire de cette capacité de travail phénoménale a été la cohérence systématique avec laquelle Coseriu a développé ses idées. Responsable des manuscrits laissés par le professeur et déposés aux Archives Coseriu de I'Université de Tübingen, Reinhard Meisterfeld observe que cette cohérence que l'on retrouve dans les écrits cosériens transparaît également dans les manuscrits :

Après avoir examiné des dizaines, voire des centaines de manuscrits cosériens, je peux affirmer en toute netteté que leur lecture nous fait découvrir la même cohérence, la même logique de l'argumentation, la même systématicité et la même profondeur analytique auxquelles les écrits publiés nous avaient déjà habitués. De plus, il est un fait connu qu'Eugenio Coseriu n'a jamais été obligé de retirer ou de moduler ses idées fondamentales, et ce, pendant plus d'un demi-siècle d'activité scientifique ! Même pour les détails placés 'en marge' de sa doctrine, les cas où il ressentait le besoin de 'toucher' à son texte étaient extrêmement rares. (BOJOGA, 2007, 130).

Dans le domaine de la philologie romane, une telle constance est exceptionnelle. Si Meisterfeld éviter de donner des exemples ou des noms concrets, force est de constater qu'il n'y a pas beaucoup de linguistes dont la communauté académique ait validé les opinions comme faisant partie d'une doctrine vraiment originale. Et même dans ces cas-là, la plupart des conceptions novatrices concernent, en règle générale, un seul domaine de la linguistique. La particularité des idées cosériennes consiste non seulement dans le fait qu'elles s'attaquent à de diverses branches de la linguistique (comme, par exemple, la typologie linguistique, la sémantique lexicale ou la linguistique du texte) mais aussi dans le fait qu'elles convergent toutes vers une vision unitaire, extrêmement synthétique du langage. Aussi certains exégètes de l'œuvre de Coseriu n’hésitent-ils à regrouper ses théories sous le nom de "linguistique réaliste" ou de "structuralisme dynamique", tandis que 
d'autres exégètes préfèrent opter pour des noms tels que «néo-traditionalisme » ou "intégralisme linguistique".

Ce qui est certain, c'est que les origines de la démarche analytique et synthétique d'Eugenio Coseriu sont à chercher dans cette extraordinaire capacité de travail. Dans son cas toutefois, affirme Kabatek, ce don s'alliait à une logique imbattable et à une mémoire exceptionnelle:

Eugenio Coseriu avait une mémoire prodigieuse, ce qui était un véritable don divin. Due probablement à son patrimoine génétique, elle était également une conséquence de son entraînement assidu. Les écrits en plusieurs langues étrangères semblaient 'occuper' une bonne partie de cette mémoire-là. Comme on le sait, il adorait parler plusieurs langues à la fois : il pouvait parler espagnol ou allemand dans les halls de l'Université et, à un moment donné, s'arrêter pour échanger deux mots en russe avec untel ou faire un petit commentaire en suédois ou bien, converser en croate avec le serveur qui travaillait au restaurant où l'on mangeait à l'époque. Avec une prononciation impeccable, Coseriu récitait des poèmes de même que de longs fragments en prose en tchèque, en suédois, en grec, en serbocroate, en catalan ou en anglais, pour ne plus mentionner ses compétences en allemand, en français, en espagnol, en italien ou en roumain. (KABATEK, 2020, p. 16)

Quant à ses capacités d'abstraction, Kabatek les place sous le signe d'une "dialectique presque médiévale [...] associée, dans sa présentation, à une connaissance approfondie des principes de la rhétorique, assimilés déjà à l'époque où il vivait encore en Roumanie" (BOJOGA, 2005, p. 18). Ces deux vertus, à savoir la mémoire infaillible et la nature logique, auraient été suffisantes, à elles seules, pour permettre à Coseriu de se forger un nom dans n'importe quel domaine, non seulement en linguistique.

La particularité de l'héritage cosérien découle de son énorme capacité de travail. II y avait quelque chose de surhumain même dans sa manière de travailler sans arrêt. II ne s'agissait pas d'une recherche faustienne de la transgression des frontières de l'humain mais plutôt d'un devoir à remplir, d'un projet à accomplir en toute lucidité, d'une construction théorique à édifier. À la manière d'Hegel, Coseriu tenait le travail, tout comme le langage, pour l'une des données fondamentales de l'être humain: si le travail concernait l'être biologique, le langage, lui, caractérisait l'être réflexif. (BOJOGA, 2005, p. 18)

L'unicité de Coseriu, selon Kabatek, réside de la rencontre si heureuse de ces trois composantes.

Des personnes à posséder une mémoire, une logique ou une capacité de travail semblables à celles de Coseriu, il y en a, peut-être. Mais il est très rare qu'on rencontre une personne qui présente toutes ces caractéristiques

Eutomia, Recife, 30(1): 1-27, Dez. 2021 
à la fois. C'est ce qui fait que Coseriu soit une personne si spéciale, si extraordinaire. C'est aussi ce qui lui a valu sa grande renommée dans le domaine de la science. Enfin, c'est ce qui a impressionné tant de générations de disciples au point de le suivre et de diffuser ses idées dans les universités des quatre coins du monde. (BOJOGA, 2005, p. 19)

\section{Références}

ALBRECHT, John. El paradigma incompleto de Eugenio Coseriu: tarea pendiente para la tercera generación. Odisea. Revista de estudios ingleses. Numero extraordinario, n. 3, 2003, p. 41-54.

BOJOGA, Eugenia. E. Coşeriu a aprins în noi flacăra cunoaşterii. Entretien avec le professeur Rudolf Windisch de l' Université de Rostock, Allemagne. Contrafort (République de Moldavie) VIII, n. 3-6, 2001, p. 31-32.

BOJOGA, Eugenia. Prof. E. Coseriu a inaugurat o panorama vasta de studii la Universitatea din Tübingen. Entretien avec le professeur Wolf Dietrich de I'Université de Münster, Allemagne, Limba Romana (République de Moldavie) n. 4-8, 2001a, p. 80-85.

BOJOGA, Eugenia. "Faptul de a-I cunoaşte pe E. Coşeriu a fost ca o provocare pentru mine". Entretien avec la professeur Ana Agud de I' Université de Salamanque, Espagne, Contrafort, n. 10-11, 2003, p. 31-32.

BOJOGA, Eugenia. "L'héritage d'Eugenio Coseriu", Eugenia Bojoga en dialogue avec le professeur Johannes Kabatek de I'Université de Tübingen, Allemagne. Contrafort, n. 11-12, 2005, p. 17-27.

BOJOGA, Eugenia. "Le principe de la tradition nous oblige à transmettre l'enseignement reçu d'Eugenio Coseriu aux générations à venir », Eugenia Bojoga en dialogue avec $M$. Reinhrad Meisterfeld. Sud-Est. Revistă trimestrială de artă, cultură şi civilizație (République de Moldavie), n. 3, 2007, p 130-143.

BOJOGA, Eugenia. Dacă studiile fundamentale ale lui E. Coşeriu ar fi fost traduse la vremea lor în engleză, astăzi lingvistica ar fi arătat cu totul altfel. Entretien avec le professeur Horst Geckeler de I'Université de Münster. Limba română (République de Moldavie), XX, n. 9-10, 2010, p. 17-24.

BOJOGA, Eugenia. Întâlnirea cu Eugeniu Coşeriu a fost decisivă pentru tot restul vieții mele. Entretien avec le professeur Nelson Cartagena de l'Université de Heidelberg, Allemagne.

Limba Română, n. 11-12, 2011, p. 92-104.

BOJOGA, Eugenia. "Am înființat editura din dorința de a-i publica studiile lui Eugeniu Coșeriu". E. Bojoga en dialogue avec M. Gunter Narr, Tübingen, Allemagne. Observator Cultural (Roumanie), n. 585, 2011a, p. 11-12.

BORCILĂ, Mircea. Eugeniu Coşeriu şi orizonturile lingvisticii. Revista de lingvistică şi ştiință literară (République de Moldavie), n. 5, 1996, p. 35-47.

Eutomia, Recife, 30(1): 1-27, Dez. 2021 
COSERIU, Eugenio. Teoría del lenguaje y lingüística general. Madrid: Gredos 1967.

GOLUBZOWA, Ludimilla. Mein langer Weg zu Eugenio Coseriu oder Über die Erfahrungen einer Seiteneinsteigerin. In: MURGUÍA, Adolfo (ed.). Sprache und Welt, Festgabe für Eugenio Coseriu zum 80. Geburtstag. Tubingen, Gunter Narr Verlag, 2002, p. 71-78.

KABATEK, Johannes; MURGUÍA, Adolfo. "Die Sachen sagen, wie sie sind..." Eugenio Coseriu im Gespräch. Tübingen: Gunter Narr 1997.

KABATEK, Johannes. Eugeniu Coseriu. Pagini de exegeză și de reconstrucție biografică. Ediție îngrijită de Cristina Bleorțu. Iași, Institutul European, 2020.

QUIJANO, Claudia Mejia. Le cours d'une vie. Portrait diachronique de Ferdinand de Saussure. Nantes, Francia: Editions Cécile Défaut, 2008.

MUNTEANU, Eugen. Eugeniu Coşeriu despre idealul paidetic. Cronica (Roumanie), n. 11, 1992, p. 1-15.

TRABANT, Jürgen. Akroamatische Aspekte der Coseriuschen Lehre. In: SCHLIEBEN-LANGE, Brigitte; TRABANT, Jürgen; WEYDT, Harald. Eugenio Coseriu: Die Sachen sagen, wie sie sind. Tübingen: Universität Tübingen 1997, p. 18-22.

TRABANT, Jürgen. Vor-Bemerkungen, dreißig Jahre danach. In: COSERIU, Eugenio. Geschichte der Sprachphilosophie. Tübingen. Basel, A. Francke Verlag, 2003, p. XI-XX.

THUN, Harald. Faire école. In: GECKELER, Horst; SCHLIEBEN-LANGE, Brigitte; TRABANT, Jürgen; WEYDT, Harald (eds.). Logos semantikos. Studia linguistica in honorem Eugenio Coseriu, v. III. Berlin: Gruyter; Madrid: Gredos, 1981. p. 325-348.

\footnotetext{
${ }^{i}$ Professeur en linguistique générale à l'Université Faculte de Lettres de I' Université Babes-Bolyai (ClujNapoca, Roumanie). Elle est titulaire d'un doctorat en linguistique et ses recherches portent sur I'historiographie linguistique, la linguistique et la sociolinguistique romanes.

ORCID : http://orcid.org/0000-0001-9821-9729.

E-mail : eugenia.bojoga@gmail.com.
}

Recebido em 24/11/21

Aprovado em 02/01/22

Todo conteúdo da Revista Eutomia está sob a Licença Creative Commons Atribuição 4.0 Internacional. 